Supplement of

\title{
The Canadian atmospheric transport model for simulating greenhouse gas evolution on regional scales: GEM-MACH-GHG v.137-reg
}

Jinwoong Kim, Saroja M. Polavarapu, Douglas Chan, and Michael Neish

Climate Research Division, Environment and Climate Change Canada, Toronto, Ontario, M3H 5T4, Canada

Correspondence to: Jinwoong Kim (Jinwoong.kim@canada.ca) 
Table S1: Root-mean squared error of zonal wind (UU; $\left.\mathrm{m} \mathrm{s}^{-1}\right)$, wind speed (UV; $\left.\mathrm{m} \mathrm{s}^{-1}\right)$, geopotential height (GZ; dam) and temperature (TT; K) at 200, 500, 850 and $1000 \mathrm{hPa}$ from GLB90, GLB45 and LAM experiments, based on comparison 24-h forecasts against North American radiosondes for July and December 2015.

\begin{tabular}{|c|c|c|c|c|c|c|c|}
\hline \multicolumn{4}{|c|}{ July } & \multicolumn{4}{c|}{ December } \\
\hline UU & GLB90 & GLB45 & LAM & UU & GLB90 & GLB45 & LAM \\
\hline $\mathbf{2 0 0}$ & 4.26 & 4.16 & 4.09 & $\mathbf{2 0 0}$ & 4.15 & 4.06 & 4.14 \\
\hline $\mathbf{5 0 0}$ & 2.7 & 2.76 & 2.79 & $\mathbf{5 0 0}$ & 3.41 & 3.42 & 3.42 \\
\hline $\mathbf{8 5 0}$ & 2.47 & 2.42 & 2.6 & $\mathbf{8 5 0}$ & 2.92 & 2.94 & 3.07 \\
\hline $\mathbf{1 0 0 0}$ & 2.36 & 2.31 & 2.55 & $\mathbf{1 0 0 0}$ & 2.33 & 2.29 & 2.38 \\
\hline UV & GLB90 & GLB45 & LAM & UV & GLB90 & GLB45 & LAM \\
\hline $\mathbf{2 0 0}$ & 4.4 & 4.2 & 4.03 & $\mathbf{2 0 0}$ & 4.26 & 4.13 & 4.2 \\
\hline $\mathbf{5 0 0}$ & 2.74 & 2.77 & 2.74 & $\mathbf{5 0 0}$ & 3.56 & 3.53 & 3.54 \\
\hline $\mathbf{8 5 0}$ & 2.53 & 2.46 & 2.54 & $\mathbf{8 5 0}$ & 3.03 & 2.86 & 2.92 \\
\hline $\mathbf{1 0 0 0}$ & 2.18 & 2.09 & 2.28 & $\mathbf{1 0 0 0}$ & 2.31 & 2.32 & 2.2 \\
\hline GZ & GLB90 & GLB45 & LAM & GZ & GLB90 & GLB45 & LAM \\
\hline $\mathbf{2 0 0}$ & 2.6 & 2.06 & 1.43 & $\mathbf{2 0 0}$ & 1.76 & 1.6 & 1.56 \\
\hline $\mathbf{5 0 0}$ & 0.86 & 0.82 & 0.81 & $\mathbf{5 0 0}$ & 1.1 & 1.05 & 1.1 \\
\hline $\mathbf{8 5 0}$ & 0.89 & 0.79 & 0.75 & $\mathbf{8 5 0}$ & 0.86 & 0.8 & 0.8 \\
\hline $\mathbf{1 0 0 0}$ & 0.9 & 0.84 & 0.9 & $\mathbf{1 0 0 0}$ & 1.04 & 0.97 & 0.91 \\
\hline TT & GLB90 & GLB45 & LAM & TT & GLB90 & GLB45 & LAM \\
\hline $\mathbf{2 0 0}$ & 1.19 & 1.11 & 1.08 & $\mathbf{2 0 0}$ & 1.36 & 1.33 & 1.35 \\
\hline $\mathbf{5 0 0}$ & 1.06 & 0.92 & 0.82 & $\mathbf{5 0 0}$ & 1.03 & 1.02 & 1.02 \\
\hline $\mathbf{8 5 0}$ & 1.24 & 1.2 & 1.17 & $\mathbf{8 5 0}$ & 1.53 & 1.5 & 1.45 \\
\hline $\mathbf{1 0 0 0}$ & 2.33 & 2.03 & 1.72 & $\mathbf{1 0 0 0}$ & 1.78 & 1.74 & 1.65 \\
\hline
\end{tabular}

